\title{
Comment on the Importance of Documenting Drug Solubility Measurement Methodology, Including Characterizing the Physical Form of the Solute, for Meaningful Understanding, Interpretation, and Reporting of Solubility Data
}

\author{
William E. Brown ${ }^{1}$, Vivian A. Gray², Johannes Krämer³, John W. Mauger ${ }^{4}$, and Kevin S. Warner ${ }^{5}$ \\ ${ }^{1}$ USP, Rockville, MD, USA. \\ ${ }^{2}$ V.A. Gray Consulting, Hockessin, DE, USA. \\ ${ }^{3}$ Disso GmbH, Homburg, Germany. \\ ${ }^{4}$ Department of Pharmaceutics and Pharmaceutical Chemistry, University of Utah College of Pharmacy, Salt Lake City, UT, USA. \\ ${ }^{5}$ Alucent Biomedical, Salt Lake City, UT, USA.
}

T his commentary continues the discussion initiated in a previous article entitled "Comment on the Importance of Data Transparency, Openness, and Reproducibility in Dissolution Science and Technology" (1). As a follow-up, this commentary more specifically provides a scan of current practices in the literature that are applicable to solubility data reported in Dissolution Technologies, with the goal of enhancing reproducibility and meaningful interpretations of these data.

Any meaningful understanding, interpretation, and reporting of a solubility measurement for a drug compound depends on accurate and reliable experimental values. In turn, accurate and reliable experimental values are influenced by experimental conditions and methodology as well as the properties of the solute and solvent (2).

Given the current emphasis of the scientific community on promoting transparency, openness, and reproducibility, this commentary is focused on the importance of precisely and completely documenting the methodology used to measure the solubility for drug compounds and the related issue of characterizing and documenting the physical form of the drug solute (3).

Accurate and reliable solubility measurements depend on the following (2):

- Control of measurement conditions (e.g., temperature and temperature control, agitation conditions, incubation time);
- Sample preparation (e.g., centrifuge or filter aliquots of slurries, then immediately dilute vehicle in appropriate solvent for highperformance liquid chromatography [HPLC] or other method of analysis);

- $\quad$ Properties of the solubility media (e.g., pH, ionic strength, added surfactants, co-solvents); and

- Physicochemical properties of the drug substance (e.g., surface area, ionization chemistry, crystal form).

Crystal structure in particular can have a direct effect on the solubility of the solid. Conversion of a metastable phase into a more stable phase via solution-mediated changes can occur (4).

Trends in the solubility of drug polymorphs have been reviewed (5). One example of the impact of the physical form of the drug on solubility was reported for ritonavir, where two crystal forms differ substantially in their solubility properties, which, in turn, creates challenges for bulk drug manufacture and formulation $(6,7)$. Another example involves rifaximin, an antibiotic intended for local action in the gastrointestinal tract, where even small amounts of the more soluble amorphous form can alter the bioavailability and pharmacological properties of this poorly absorbed drug (8).

With this background in mind, documenting the methodology for solubility measurements, including characterizing the physical form of the drug solute, 
is relevant to the accuracy, reproducibility, and interpretation of the solubility data. Methods for determining solubility have been reviewed by several authors (2, 9-13). Murdande et al. provide useful and practical precautions to be taken when measuring solubility, such as (11):

- Ensuring high chemical purity of solute and solvent;

- Selecting satisfactory techniques for complete separation of the saturated solution from undissolved particles;

- Employing appropriate analytical methods to quantify drug concentration in solution;

- Ability to detect decomposition products;

- Temperature control;

- Measurement of $\mathrm{pH}$ of the solution at the end of the equilibration period for aqueous solutions; and

- Analyzing and characterizing the excess solid separated from the saturated solution to determine whether any changes in solid phase may have occurred relative to the initial starting material.

Murdande et al also provides detailed procedures for solubility measurement of crystalline and amorphous forms (11). Dressman et al. have given special attention to measuring drug solubility in the gastrointestinal tract (14). A review concerning the estimation of the solubility of metastable polymorphs is available (15).

Higuchi et al. addressed special problems with measuring the solubility of very sparingly soluble drug substances, which is particularly problematic in the presence of impurities, and proposed an experimental approach to overcome this problem (16). A standardized protocol using the Higuchi method to measure the equilibrium solubility measurement of compounds with low dissolution rate has more recently been validated $(16,17)$. Methods for characterizing the physical form of the drug substance include microscopy, spectroscopy, X-ray diffraction, and thermal analysis. These and other methods have been reviewed (18-21). It should be noted that more than one method may be necessary to fully characterize the physical form of a drug substance, as was the case for ritonavir (7).
This commentary has provided a brief scan of the literature concerning methods to measure solubility and characterize the physical form of the drug. This sample of relevant literature emphasizes the need to carefully control experimental conditions for solubility studies and provides the rationale for characterizing the physical form of the drug.

Therefore, reviewers and readers of solubility data submitted for publication in Dissolution Technologies should have access to complete and precise methodology used for any solubility measurement, including characterization of the physical form of the drug solute before and after the solubility experiment. Because in vitro drug dissolution rates are directly related to drug solubility $(22,23)$, it follows that a similar argument can be applied to the measurement and reporting of dissolution data.

Any lack of critical information related to solubility measurement methodology and characterization of the physical form of the drug solute creates questions about the interpretation and reproducibility of these data, with reproducibility being of particular importance because, as emphasized by McNutt (24), "reproducing an experiment is one important approach that scientists use to gain confidence in their conclusions".

\section{CONFLICT OF INTEREST}

The authors disclosed no conflicts of interest related to this article.

\section{REFERENCES}

1. Mauger, J. W. Comment on the importance of data transparency, openness, and reproducibility in dissolution science and technology. Dissolution Technol. 2018, 25, 6-7. DOI: 10.14227/ DT250218P6.

2. <1236> Solubility Measurements. The United States Pharmacopeia and National Formulary USP 43-NF 38, 2nd Supplement, The United States Pharmacopeial Convention, Inc.: Rockville, MD, 2019.

3. Nosek, B. A.; Alter, G.; Banks, G. C.; Borsboom, D.; Bowman, S. D.; Breckler, S. J.; Buck, S.; Chambers, C. D.; Chin, G.; Christensen, G.; Contestabile, M.; Dafoe, A.; Eich, E.; Freese, J.; Glennerster, R.; Goroff, D.; Green, D. P.; Hesse, B.; Humphreys, M.; Ishiyama, J.; Karlan, D.; Kraut, A.; Lupia, A.; Mabry, P.; Madon, T.; Malhotra, N.; Mayo-Wilson, E.; McNutt, M.; Miguel, E.; Paluck, E. L.; Simonsohn, U.; Soderberg, C.; Spellman, B. A.; Turitto, J.; VandenBos, G.; Vazire, S.; Wagenmakers, E. J.; Wilson, R; Yarkoni, $\mathrm{T}$. Promoting an open research culture: author guidelines for journals could help to promote transparency, openness, and 
reproducibility. Science 2015, 348, 1422-1425. DOI: 10.1126/ science.aab2374.

4. Brittain, H. G. Thermodynamic vs. kinetic solubility: knowing which is which. American Pharmaceutical Review website. (April 29, 2014). https://www.americanpharmaceuticalreview. com/Featured-Articles/160452-Thermodynamic-vs-KineticSolubility-Knowing-Which-is-Which/(accessed July 12, 2019).

5. Pudipeddi, M.; Serajuddin, A. T. M. Trends in solubility of polymorphs. J. Pharm. Sci. 2005, 94, 929-939. DOI: 10.1002/ jps.20302.

6. Chemburkar, S. R.; Bauer, J.; Deming, K.; Spiwek, H.; Patel, K.; Morris, J.; Henry, R.; Spanton, S.; Dziki, W.; Porter, W.; Quick, J.; Bauer, P.; Donaubauer, J.; Narayanan, B. A.; Soldani, M.; Riley, D.; McFarland, K. Dealing with the impact of ritonavir polymorphs on the late stages of bulk drug process development. Org. Process Res. Dev. 2000, 4, 413-417. DOI: 10.1021/op000023y.

7. Bauer, J.; Spanton, S.; Henry, R.; Quick, J.; Dziki, W.; Porter, W.; Morris, J. Ritonavir: An extraordinary example of conformational polymorphism. Pharm. Res. 2001, 18, 859-866. DOI: 10.1023/A:1011052932607.

8. Blandizzi, C.; Viscomi, G.C.; Scarpignato, C. Impact of crystal polymorphism on the systemic bioavailability of rifaximin, an antibiotic acting locally in the gastrointestinal tract, in healthy volunteers, Drug. Des. Dev. Ther. 2015, 9, 1-11. DOI: 10.2147/ DDDT.S72572.

9. Sou, T.; Bergström, C. A. S. Automated assays for thermodynamic (equilibrium) solubility determination. Drug Discovery Today: Technol. 2018, 27, 11-19. DOI: 10.1016/j.ddtec.2018.04.004.

10. Black, S.; Dang, L.; Liu, C.; Wei.,H. On the measurement of solubility. Org. Process. Res. Dev. 2013, 17, 486-492. DOI: 10.1021/op300336n.

11. Murdande, S. B.; Pikal, M. J.; Shanker, R. M.; Bogner, R. H. Aqueous solubility of crystalline and amorphous drugs: challenges in measurement. Pharm. Dev. Technol. 2011, 16, 187-200. DOI: 10.3109/10837451003774377.

12. Alsenz, J.; Kansy, M. High throughput solubility measurement in drug discovery and development. Adv. Drug Delivery Rev. 2007, 59, 546-567. DOI: 10.1016/j.addr.2007.05.007.

13. Adveef, A.; Fuguet, E.; Llinàs, A.; Ràfols, C.; Bosch, E.; Völgyi, G.; Verbić, T.; Boldyreva, E.; Takács-Novák, K. Equilibrium solubility measurment of ionizable drugs - consensus recommendations for improving data quality. ADMET. 2016, 4, 117-178. DOI: 10.5599/admet.4.2.292.

14. Dressman, J. B.; Vertzoni, M.; Goumas, K.; Reppas, C. Estimating drug solubility in the gastrointestinal tract. Adv. Drug Delivery Rev. 2007, 59, 591-602. DOI: 10.1016/jaddr.2007.05.009.

15. Nicoud, L.; Licordari, F.; Myerson, A. S. Estimation of the solubility of metastable polymorphs: a critical review. Cryst. Growth Des. 2018, 18, 7228-7237. DOI: 10.10121/acs.cgd.8b01200.

16. Higuchi, T.; Shih, F-M. L.; Kimura, T.; Rytting, J. H. Solubility determination of barely aqueous-soluble organic solids. J. Pharm. Sci. 1979, 68, 1267-1272. DOI: 10.1002/jps.2600681019.

17. Takács-Novák, K.; Urac, M.; Horváth, P.; Völgyi, G.; Anderson, B. D.; Avdeef, A. Equilibrium solubility measurement of compounds with low dissolution rate by Higuchi's facilitated dissolution method. A validation study. Eur. J. Pharm. Sci. 2017, 106, 133141. DOI: 10.1016/j.ejps.2017.05.064.

18. Brittain, H. G., Ed.; Polymorphism in Pharmaceutical Solids, 2nd. ed; Drugs and the Pharmaceutical Sciences, volume 192; Informa Healthcare USA, Inc.; New York, N.Y. ; 1999, pp 318-435.

19. Healy, A. M.; Worku, Z. A.; Kumar, D.; Madi, A. M. Pharmaceutical solvates, hydrates and amorphous forms: a special emphasis on cocrystals. Adv. Drug Delivery Rev. 2017, 117, 25-46. DOI: 10.1016/j.addr.2017.03.002.

20. Suryanarayanan, R.; Byrn, S. Characterization of the solid state. Adv. Drug Delivery Rev. 2001, 48, 1-136. DOI: 10.1016/S0169409X(01)00096-5.

21. Harris, R. K, NMR studies of organic polymorphs \& solvates. Analyst. 2006, 131, 351-373. DOI: 10.1039/b516057j.

22. Noyes, A. A.; Whitney, W. R. The rate of solution of solid substances in their own solutions. J. Am. Chem. Soc. 1897, 19, 930-934. DOI: 10.1021/ja02086a003.

23. Amidon, G. L.; Lennernäs, H.; Shah, V. P.; Crison, J. R. A theoretical basis for a biopharmaceutic drug classification: the correlation of in vitro drug product dissolution and in vivo bioavailability. Pharm. Res. 1995, 12, 413-420. DOI:10.1023/A:1016212804288.

24. McNutt, M. Reproducibility. Science 2014, 343, 229. DOI:10.1126/science.1250475. 